\title{
Tolerance Induced by (S)-3,5-Dihydroxyphenylglycine Postconditioning is Mediated by the PI3K/Akt/GSK3 $\beta$ Signalling Pathway in an In Vitro Model of Cerebral Ischemia
}

\author{
Elisabetta Gerace, ${ }^{\dagger}$ Tania Scartabelli, $^{\dagger}$ Domenico E. Pellegrini-Giampietro ${ }^{\star}$ and Elisa Landucci ${ }^{* *}$ \\ Department of Health Sciences, Section of Clinical Pharmacology and Oncology, University of Florence, Florence, Italy
}

\begin{abstract}
Ischemic postconditioning (PostC) is an endogenous neuroprotective strategy for cerebral ischemia induced by low activation of glutamate receptors. We have previously shown that the application of the mGluR1/5 agonist (S)-3,5-dihydroxyphenylglycine (DHPG) $5 \mathrm{~min}$ after $30 \mathrm{~min}$ of oxygen and glucose deprivation (OGD) reduces CA1 damage in organotypic hippocampal slices by activating the PI3K-Akt signalling pathway. In order to extend these data, we analysed the production of reactive oxygen species (ROS) and the glycogen synthase kinase $3 \beta$ (GSK3 $\beta$ ) signalling pathway. Our results show that DHPG PostC was associated with a reduction in the formation of ROS that is massively increased $24 \mathrm{~h}$ after OGD exposure. This reduction was prevented by the PI3K inhibitor LY294002, indicating that there is a link between the PI3K/Akt pathway and the formation of ROS in the protective mechanisms of PostC. DHPG PostC also induces a transient increased in GSK3 $\beta$ phosphorylation and inactivation that is followed by nuclear accumulation of $\beta$-catenin, that probably lead to the upregulation of neuroprotective genes. Our results propose GSK3 $\beta$ as new target for neuroprotection, therefore, we verified that the two GSK3 $\beta$ inhibitors N-(3-Chloro-4-methylphenyl)-5-(4-nitrophenyl)-1,3,4-oxadiazol-2-amine (TC-G 24) and $\mathrm{LiCl}$ are neuroprotective agents in OGD and also can be used as PostC agents. $\odot 2020$ IBRO. Published by Elsevier Ltd. All rights reserved.
\end{abstract}

Key words: DHPG postconditioning, oxygen and glucose deprivation, PI3K/Akt/GSK3ß signaling pathway, organotypic hippocampal slices, Lithium chloride.

\section{INTRODUCTION}

Ischemic postconditioning (PostC) has been established as a novel neuroprotective strategy against ischemic stroke and is defined as a single or a series of brief interruptions in the cerebral blood flow supply performed immediately after a severe ischemic insult (Zhao et al., 2012). However, the molecular mechanisms responsible for neuroprotection induced by PostC remain largely unclear. In a previous paper of our laboratory, we have showed that it is possible to evoke a neuroprotective PostC response in organotypic hippocampal slices exposed to $30 \mathrm{~min}$ OGD by applying, 5 min later, a rela-

\footnotetext{
${ }^{*}$ Corresponding author. Address: Department of Health Sciences, Clinical Pharmacology and Oncology Unit, University of Florence, Viale G. Pieraccini 6, 50139 Florence, Italy. Fax: + 39-055-2751093. E-mail address: elisa.landucci@unifi.it (E. Landucci).

Elisabetta Gerace and Tania Scartabelli contributed to the study equally.

\$ Domenico E. Pellegrini-Giampietro and Elisa Landucci contributed equally.

Abbreviations: CREB, CAMP response element-binding; DHPG, (S)3,5-dihydroxyphenylglycine; GSK3 $\beta$, glycogen synthase kinase $3 \beta$; OGD, oxygen and glucose deprivation; PostC, postconditioning; PreC, Preconditioning; ROS, reactive oxygen species; TC-G 24, N-(3-Chloro4-methylphenyl)-5-(4-nitrophenyl)-1,3,4-oxadiazol-2-amine.
}

tively low dose of the mGluR1/5 agonist 3,5dihydroxyphenylglycine (DHPG) (Scartabelli et al., 2008). This neuroprotection is mediated by the activation of the mGlu1/mGlu5-PI3K-Akt signaling pathway and can be completely abolished by inhibitors of PI3K and Akt activity (Scartabelli et al., 2008). However, our understanding of the mechanistic pathways linking DHPG PostC and neuroprotection remains incomplete.

It has been largely demonstrated that after an ischemic insult, the production of reactive oxygen species (ROS) increases and leads to tissue damage (Liu et al., 2003). Despite this, ROS have been paradoxically implicated as an important signaling component in neuroprotective pathways activated by preconditioning PreC (Nie et al., 2006), PostC (Tsutsumi et al., 2007) and remote ischemic postconditioning (RPostC) (Wang et al., 2011). Oxidative stress that follows reperfusion is related to the enormous generation of ROS and is considered injurious to cell functions and mitochondria (Song et al., 2013). The protective effects of PostC in ische$\mathrm{mia} /$ reperfusion injury is not only associated with inhibition of inflammation and apoptosis (Xing et al., 2008a,b), but also with attenuation of oxidative stress (Wang et al., 2008; Ren et al., 2008). 
The PI3K/Akt pathway has been indicated to play a critical role in survival in PreC (Gerace et al., 2012b; Landucci et al., 2016) and PotsC (Ye et al., 2012). Zhou and colleagues (Zhou et al., 2011) have also shown that phosphorylation (ser 9) and subsequent inactivation of glycogen synthase kinase $3 \beta$ (GSK3 $\beta$ ) via the PI3K/Akt pathway is an important element in the neuroprotective effects of delayed ischemic PostC against global cerebral ischemia. GSK3 $\beta$ is not only a downstream target of Akt, but is also involved in the Wnt pathway (Cook et al., 1996). GSK-3 inactivation has been proposed as a mechanism to promote neuronal survival (Liang and Chuang, 2007). In fact, when GSK3 $\beta$ is inactivated, it leads to $\beta$ catenin accumulation and translocation from the cytosol to the nucleus (Ding et al., 2000). $\beta$-Catenin mediates anti-apoptotic effects by regulating the expression of several important anti-apoptotic genes such as fibronectin, cyclin DI, c-myc and CaMKIV (Gordon and Nusse, 2006). Increased intracellular calcium levels trigger a series of events that involve not only CaMKIV gene expression, but also cAMP response element-binding (CREB) protein phosphorylation that promote survival through the transcription of CREB-dependent genes, such as Cfos (Gallin and Greenberg, 1995). Lithium as well as other GSK-3 $\beta$ inhibitors has neuroprotective effects against neurological disorders. Delayed lithium treatment provided neuroprotection against focal (Taliyan and Ramagiri, 2016) and global ischemia (Bian et al., 2007).

The aim of the present study was to examine the hypothesis that the protection afforded by DHPG PostC is mediated via the PI3K/Akt/GSK3 $\beta$ signaling pathway in rat organotypic hippocampal slices.

\section{EXPERIMENTAL PROCEDURES}

Male and female Wistar rat were obtained from Charles River (MI, Italy). Animals were housed at $23 \pm 1^{\circ} \mathrm{C}$ under a $12 \mathrm{~h}$ light-dark cycle (lights on at 07:00) and were fed a standard laboratory diet with ad libitum access to water. Experiments and animal use procedures were in accordance with the National Institutes of Health Guide for the Care and Use of Laboratory Animals (NIH Publications No. 80-23, revised 1996).

The experimental protocols were approved by the Animal Care Committee of the Department of Health Sciences, University of Florence, in compliance with the European Convention for the Protection of Vertebrate Animals used for Experimental and Other Scientific Purposes (ETS No. 123) and the European Communities Council Directive of 2010/63/EU. The authors further attest that all efforts were made to minimize the number of animals used and their suffering.

\section{Materials}

DHPG was purchased from Abcam plc (Cambridge, UK). $\mathrm{N}$-(3-Chloro-4-methylphenyl)-5-(4-ni-trophenyl)-1,3,4-oxa diazol-2-amine (TC-G 24) was purchased from Tocris Bioscience (Bristol, United Kingdom, UK). Propidium iodide (PI), lithium chloride and resveratrol were purchased from Sigma (St Louis, MO, USA). 2-(4-Morpho
linyl)-8-phenyl-4H-1-benzopyran-4-one (LY294002) was obtained from Calbiochem (Merck Biosciences Ltd, Nottingham, UK). Tissue culture reagents were obtained from Gibco-BRL (San Giuliano Milanese, MI, Italy) and Sigma (St Louis, MO, USA).

\section{Preparation of rat organotypic hippocampal slice cultures}

Organotypic hippocampal slice cultures were prepared as previously reported (Gerace et al., 2012a). Briefly, transverse hippocampal slices $(420 \mathrm{~mm})$ were obtained from 7 to 9 days old Wistar rats and were prepared using a Mcllwain tissue chopper. The integral slices were selected and then cultured onto semiporous membrane inserts (Millicell-CM; Millipore, Italy) placed in six well tissue culture plates containing $1.2 \mathrm{ml}$ medium per well (50\% Eagle's minimal essential medium, $25 \%$ heatinactivated horse serum, $25 \%$ Hanks' balanced salt solution, $5 \mathrm{mg} / \mathrm{ml}$ glucose, $2 \mathrm{mM}$ L-glutamine, and $3.75 \mathrm{mg} / \mathrm{ml}$ amphotericin B). Slices were kept in culture for 14 days and at $37^{\circ} \mathrm{C}$ in an incubator in atmosphere of humidified air and $5 \% \mathrm{CO}_{2}$. Before experiments all slices were screened for viability by phase-contrast microscopy analysis; slices displaying signs of neurodegeneration were discarded from the study.

\section{OGD and postconditioning protocols in rat organotypic hippocampal slices}

Cerebral ischemia in vitro was mimicked by exposing hippocampal cultures to $30 \mathrm{~min}$ of oxygen and glucose deprivation (OGD) as previously reported in detail (Gerace et al., 2015). Following $30 \mathrm{~min}$ of incubation at $37^{\circ} \mathrm{C}$ in an anaerobic chamber (BioSpherix, New York, USA), the cultures were transferred to oxygenated serum-free medium and returned to the incubator under normoxic conditions. Neuronal injury was evaluated $24 \mathrm{~h}$ later. For postconditioning experiments, organotypic hippocampal slices were exposed firstly to $30 \mathrm{~min} O G D$ and $5 \mathrm{~min}$ later to $10 \mu \mathrm{M}$ DHPG for $30 \mathrm{~min}$ (Scartabelli et al., 2008).

\section{Assessment of CA1 pyramidal cell injury}

Cell injury was assessed by using the fluorescent dye propidium iodide ( $\mathrm{Pl}, 5 \mu \mathrm{g} / \mathrm{ml}$ ). Fluorescence was viewed using an inverted fluorescence microscope (Olympus IX-50; Solent Scientific, Segensworth, United Kingdom) equipped with a xenon-arc lamp, a low-power objective $(4 \times)$ and a rhodamine filter. Images were digitized using a video image obtained by a CCD camera (Diagnostic Instruments Inc., Sterling Heights, MI, United States) controlled by software (InCyt Im1TM; Intracellular Imaging Inc., Cincinnati, $\mathrm{OH}$, United States) and subsequently analysed using a morphometric analysis software Image-Pro Plus morphometric analysis software (Media Cybernetics, Silver Spring, MD, United States). In order to quantify cell death, the CA1 hippocampal subfield was identified and encompassed in a frame using the drawing function in the image 
software (ImageJ; NIH, Bethesda, USA) and the optical density of PI fluorescence was detected.

\section{Reactive oxygen species (ROS) production}

Oxidant-sensing fluorescent probe, 2,7dichlorofluorescein diacetate (DCFH2-DA) was used to detected the formation of intracellular peroxides in organotypic hippocampal slices (Liu et al., 2003). Organotypic hippocampal slices were incubated with $10 \mu \mathrm{M}$ DCFH2-DA at $37^{\circ} \mathrm{C}$ for $30 \mathrm{~min}$. Fluorescence was viewed $30 \mathrm{~min}$ later using an inverted fluorescence microscope (Olympus IX-50; Solent Scientific, Segensworth, UK) equipped with a xenon-arc lamp and a rhodamine filter (excitation 450 and $490 \mathrm{~nm}$, emission $520 \mathrm{~nm}$ ). Images were digitized using a video image obtained by a CCD camera (Diagnostic Instruments Inc., Sterling Heights, MI, USA) controlled by software (InCyt Im1; Intracellular Imaging Inc., Cincinnati, OH, USA) and subsequently analyzed using the Image-Pro Plus morphometric analysis software (Media Cybernetics, Silver Spring, MD, USA). In order to quantify ROS production, the CA1 hippocampal subfield was identified and encompassed in a frame using the drawing function in the image software (ImageJ; NIH, Bethesda, USA) and the optical density of DCFH2-DA fluorescence was detected (Boscia et al., 2006).

\section{Western blot analysis}

Western blotting experiments were performed as previously reported in (Landucci et al., 2016). Briefly,

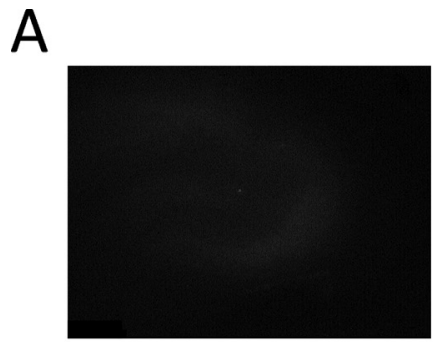

CRL

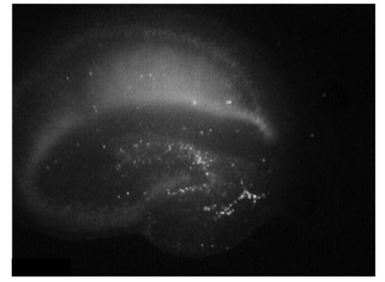

$30 \mathrm{~min}$ OGD

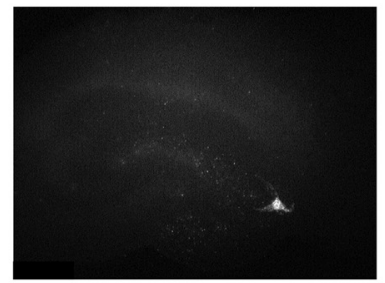

DHPG PostC

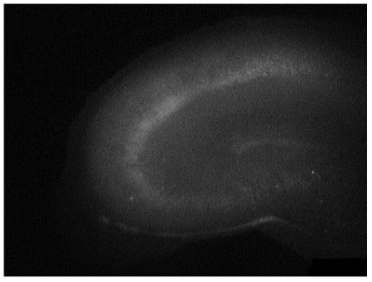

DHPG PostC + LY294002
B

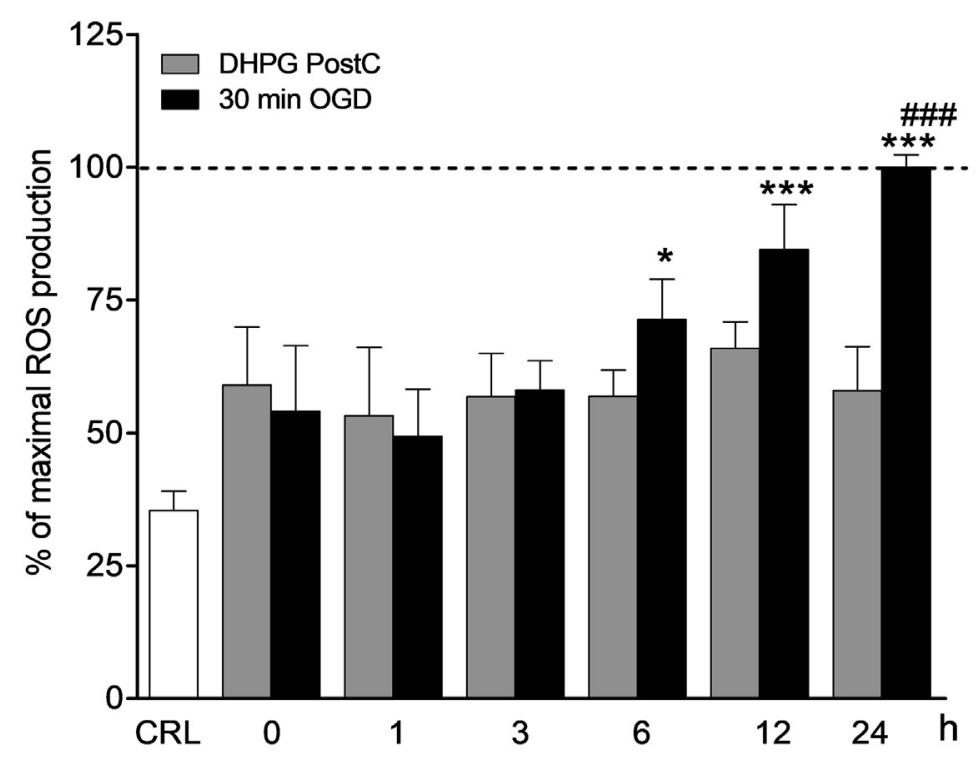

\section{C}

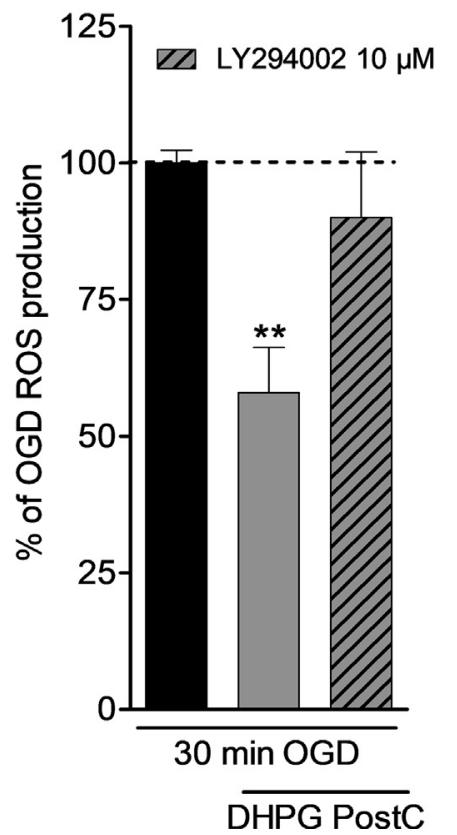

Fig. 1. DHPG PostC induces neuroprotection against OGD injury by reducing the formation of ROS through PI3K pathway. (A) Under control conditions, organotypic hippocampal slices display background DCF fluorescence. $24 \mathrm{~h}$ after DHPG PostC the slices displayed a marked reduction of neuronal injury in the CA1 subregion induced by 30 min OGD. This reduction is reverted by the incubation with LY294002 during DHPG PostC and during the subsequent $24 \mathrm{~h}$. (B) Bars represent the quantitative analysis of CA1 DCF fluorescence and show that OGD induced a timedependent increase in ROS formation. This increase is significantly reduced by DHPG PostC. (C) Quantitative analysis showing that the toxicity produced by OGD was reduced by DHPG PostC and this reduction was prevented by LY294002. Data are expressed as percentage of OGDinduced ROS production in CA1 region. Bars represent the mean \pm SEM of at least four experiments run in quadruplicate. (B) ${ }^{*} p<0.05$, ${ }^{* * *} p<0.001$ vs. CRL; ${ }^{\# \#} p<0.001$ DHPG PostC vs. OGD 24 h. (C) ${ }^{* *} p<0.01$ vs. OGD (ANOVA + Tukey's $w$ test). 
cultured slices were dissolved in 1\% SDS and protein levels were quantified using the Pierce (Rockford, IL, USA) BCA (bicinchoninic acid) Protein Assay. Lysates were resolved by electrophoresis on a 4-20\% Tris Glycine Gel (Invitrogen, San Giuliano Milanese, Italy) and transferred onto nitrocellulose membranes (Bio-Rad Laboratories, CA, USA). After blocking in 5\% non-fat dry milk, the membranes were incubated overnight at $4{ }^{\circ} \mathrm{C}$ with a polyclonal antibodies directed against $\beta$-catenin (Sigma, St Louis, MO, USA), phospho-GSK3- $\beta$ (Ser9), phosphoCREB (Ser133) and acetyl H3 (K18), (all from Cell Signaling Technology, Beverly, MA, USA) rabbit polyclonal anti GFAP (Sigma, STLOUIS, MO, USA) anti IBA (WAKO, Osaka, Japan) anti YM1 (Stemcell, Vancouver, Canada) (primary antibody dilution 1:1000). Monoclonal anti- $\beta$ actin and anti $\beta$-tubulin antibodies were used as loading controls. Anti-mouse or anti-rabbit secondary antibodies (Amersham Biosciences, UK) conjugated to horseradish peroxidase were used for immunodetection. The reactive bands were detected using chemiluminescence (ECL; Amersham Biosciences, Buckinghamshire, UK). Quantitative analysis of reactive bands was performed using the Quantity One analysis software (Bio-Rad, Hercules, CA, USA).

\section{Nuclear and cytosol isolation}

Nuclei and cytosol were isolated from organotypic hippocampal slices $1 \mathrm{~h}$ after the end of the experimental protocols as described in (Gerace et al., 2014). Organotypic slices were homogenized in a glass/glass potter with $500 \mu \mathrm{l}$ of isolation buffer (TRIS/MOPS $10 \mathrm{mM}$ EGTA/TRIS $1 \mathrm{mM}$, sucrose $200 \mathrm{mM} \mathrm{pH} \mathrm{7.4).} \mathrm{Nuclear}$ fraction was obtained after centrifugation at $600 \mathrm{~g}$ for $10 \mathrm{~min}$ and mitochondrial pellet were obtained after centrifugation at $7000 \mathrm{~g}$ for $10 \mathrm{~min}$. Supernatants resulted in cytosolic fraction. Nuclear and cytosolic protein levels were quantified using the Pierce (Rockford, IL, USA) BCA (bicinchoninic acid) Protein Assay and processed by Western Blotting.

\section{Statistical analysis}

Statistical significance of differences between DCFH2DA, propidium iodide fluorescence intensities or Western blot optical densities was evaluated by performing analysis of variance (ANOVA) followed by the Tukey's w test for multiple comparisons. Differences were considered significant for ${ }^{*} p<0.05$, ${ }^{* *} p<0.01$. Graph Pad Prism (Graph Pad Software, San Diego, CA) was used for performing all statistical analysis.

\section{RESULTS}

DHPG postconditioning induces neuroprotection by reducing ROS formation and by activating the PI3K/ Akt/GSK3 $\beta$ signaling pathway in organotypic hippocampal slices exposed to OGD

As previously reported, organotypic hippocampal slices exposed to DHPG postconditioning (DHPG PostC) showed a significant reduction of neuronal injury induced by 30 min OGD via the activation of PI3K-Akt signaling pathway (Scartabelli et al., 2008). Fig. 1 shows that DHPG PostC is also able to decrease the levels of ROS formation that has been induced by OGD, starting from $6 \mathrm{~h}$ after PostC treatment to a maximal effect $24 \mathrm{~h}$ later (Fig. 1, panel $A$ and $B$ ). This reduction is similar to what obtained by the classical ROS scavenger resveratrol $(10 \mu \mathrm{M})$, that is also able to induce neuroprotection when used in the same experimental condition of DHPG PostC (Supplementary data, Fig. 1). The decrease in ROS production provoked by DHPG PostC is prevented by the PI3K inhibitor LY294002, indicating that there is a relation between PI3K-Akt pathway activation and ROS formation in the protective mechanisms of PostC (Fig. 1, panel C).

We then examined the hypothesis that the neuroprotection induced by DHPG PostC could be mediated by GSK3 $\beta$, a downstream target of the abovementioned signaling pathway. In particular, GSK3 $\beta$ could have an important role in the regulation of $\beta$ catenin activation and translocation from cytoplasm to the nucleus, where $\beta$-catenin acts as a transcriptional coactivator, activating genes involved in cell survival and neuroprotection. To this aim, we firstly used a

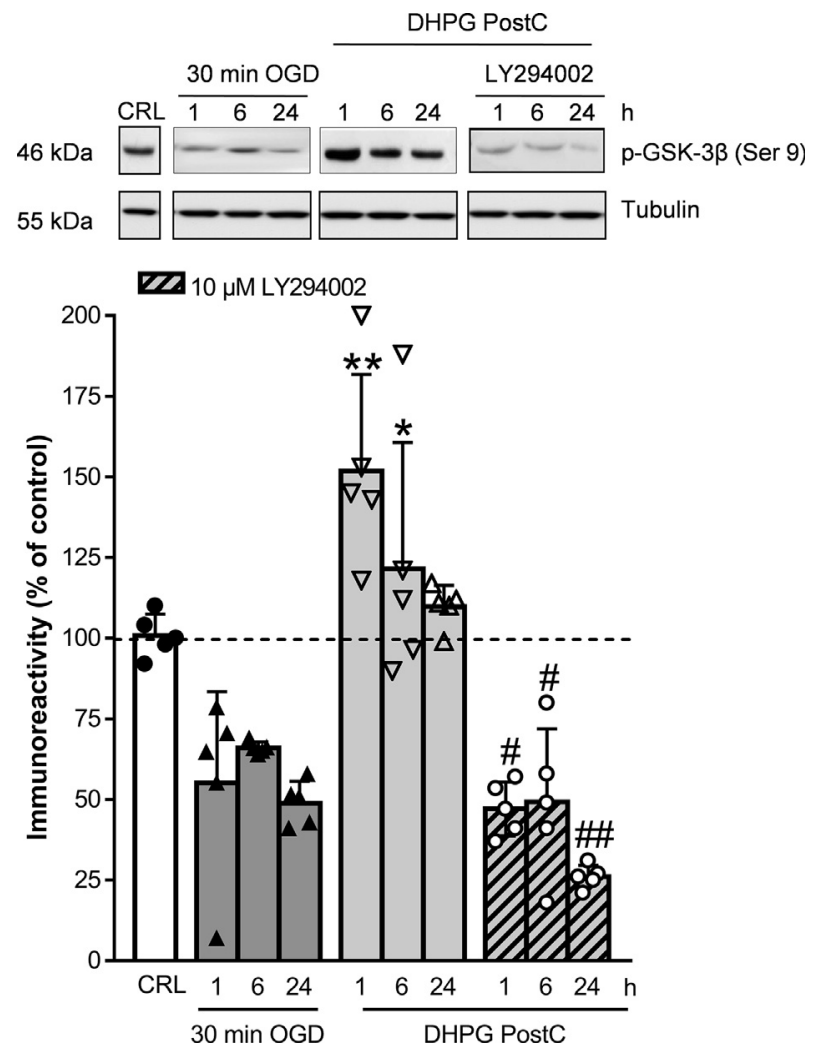

Fig. 2. DHPG PostC induces PI3K-Akt signaling pathway-dependent increase in GSK-3 $\beta$ phosphorylation. 1, 6 or $24 \mathrm{~h}$ after treatments Western blot analysis were performed to assess the levels of GSK-3 $\beta$ phosphorylation. Upper panel: representative Western blot for phosphorylated GSK-3 $\beta$ (p-GSK-3 $\beta$ ). Lower panel: quantitative analysis showing that DHPG PostC induced an increase in GSK-3 $\beta$ phosphorylation that was significantly decreased in presence of the Akt inhibitor LY294002. Data are expressed as percentage of GSK-3 $\beta$ phosphorylation in control slices. Bars represent the mean \pm SEM of at least five experiments. ${ }^{*} p<0.05$ and ** $p<0.01$ vs. CRL. ${ }^{\#} p<0.05$ and ${ }^{\#} p<0.01$ vs. 1 h DHPG PostC (ANOVA + Tukey's $w$ test). 
phospho-specific (Ser9) antibody to measure the levels of GSK3 $\beta$ after exposure to OGD or to DHPG PostC protocol. We observed that OGD induced a decrease in GSK3 $\beta$ phosphorylation at different time point (Fig. 2). On the contrary, pharmacological PostC leads to a transient increase in GSK3 $\beta$ phosphorylation that is maximal $1 \mathrm{~h}$ after treatment and returned to basal levels $24 \mathrm{~h}$ later (Fig. 2). The PostC-induced increase in GSK3 $\beta$ phosphorylation was reduced by LY294002 (Fig. 2), confirming that the activation of the survival kinase Akt is responsible for GSK3 $\beta$ inactivation.

In addition, we analyzed the cytosolic and nuclear levels of $\beta$-catenin, one of the substrates of GSK3 $\beta$, in order to determine whether it accumulates in the cytoplasm and consequently translocates to the nucleus after DHPG PostC. Our data show that OGD did not affect protein levels neither in cytosol nor in nucleus in organotypic hippocampal slices (Fig. 3). On the contrary, $1 \mathrm{~h}$ after DHPG PostC treatment we observed

\section{Cytoplasmic fraction}

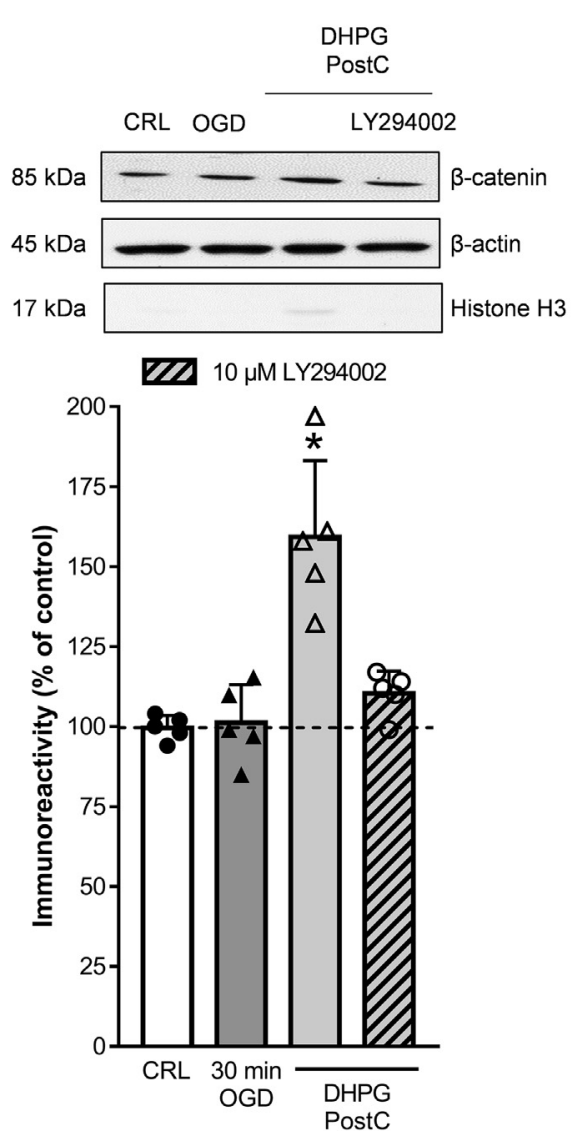

\section{Nuclear fraction}
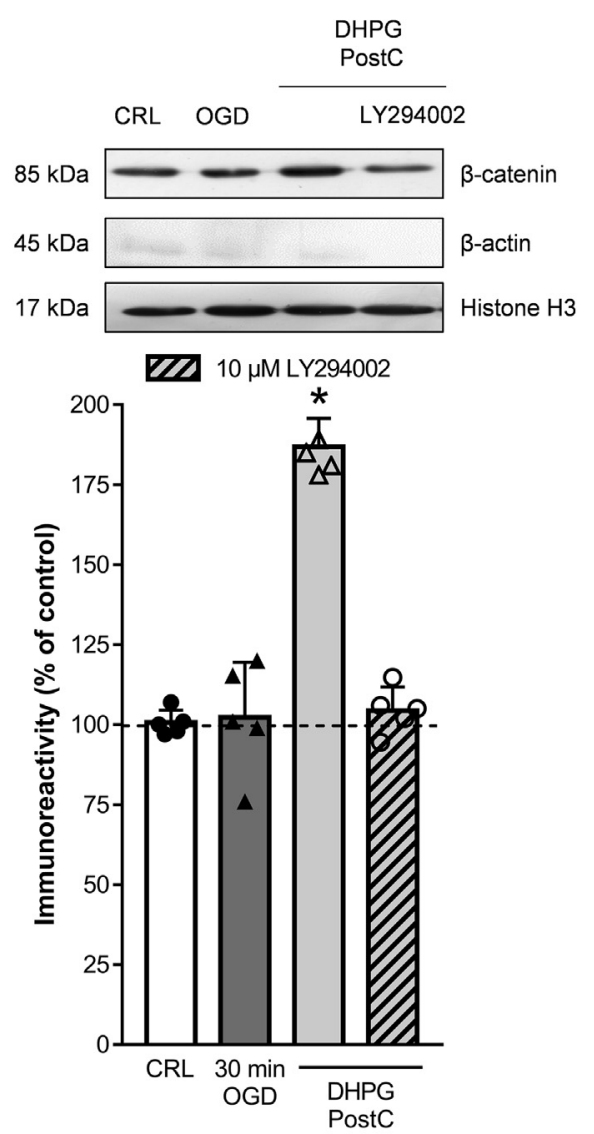

Fig. 3. DHPG PostC is associated with cytosolic and nuclear accumulation of $\beta$-catenin. Slices were exposed to 30 min OGD, DHPG PostC and DHPG PostC plus the Akt inhibitor LY294002. The level of $\beta$-catenin was determined by Western blot analysis in cytosolic (left panel) and nuclear (right panel) fractions $1 \mathrm{~h}$ after treatments. Representative Western blot for $\beta$-catenin (upper pane/) and quantitative analysis (lower panel) of immunoreactive bands showing that DHPG PostC but not OGD induced a significantly increase in $\beta$-catenin in both cytosolic and nuclear fractions. This increase is mediated by PI3K-Akt activation and is prevented by the Akt inhibitor LY294002. Data are expressed as percentage of $\beta$-catenin in control untreated slices. Data are expressed as percentage of $\beta$-catenin in control untreated slices. Bars represent the mean \pm SEM of at least five experiments. ${ }^{*} p<0.05$ vs. CRL (ANOVA + Tukey's $w$ test). a significant increase of $\beta$-catenin expression in both cytosolic and nuclear fractions that is completely reverted to the basal level by Akt inhibitor LY294002

To elucidate whether DHPG PostC could promote neuronal survival through accumulation of nuclear $\beta$ catenin, we analyzed the cAMP response elementinding protein (CREB) phosphorylation (Ser133) in our corce to OGD or to PostC alone or in PostC induces a significant increase in CREB phosphorylation that is completely prevented by the cocubation with the Akt inhibitor.

neuroprotection, the inhibitors TC-G 24 and $\mathrm{LiCl}$ as neuroprotective agents in OGD and also as PostC agents. Our results confirm our hypothesis and show that both GSK3 $\beta$ inhibitors significantly reduce the damage induced by OGD (Fig. 5, panel $A$ and $B$ ). Furthermore, slices exposed to TC-G 24 or $\mathrm{LiCl}$ for $30 \mathrm{~min}, 5 \mathrm{~min}$ after the toxic OGD insult (PostC protocol) display a significant reduction of CA1 toxicity that is similar to what obtained with DHPG PostC (Fig. 5, panel A and C), confirming and strengthening the crucial role of GSK3 $\beta$ in PostC and neuroprotection.

\section{DISCUSSION}

Ischemic PostC has been shown to be protective against ischemic injury in different organs including liver, intestine and brain (Guo et al., 2011; Liu et al., 2003; Pignataro et al., 2008). The underlying mechanisms were related to multiple factors including regulation of enzyme activity, inhibition of endoplasmic stress, activation of signaling pathways, suppression of oxidative stress and effective regulation of cerebral blood flow to the ischemic area. The present study provides findings that increase our understanding of the signal transduction mechanism of the neuroprotection afforded by pharmacological PostC $(10 \mu \mathrm{M}$ DHPG for $30 \mathrm{~min}$ ) in a rat hippocampal slice model of cerebral ischemia. In our previous work, we have demonstrated that our PostC protocol increases the phosphorylation of Akt in a transient and mGlu1/mGlu5dependent manner and that the inhibitors of PI3K and Akt activity 
completely abolish the neuroprotection induced by PostC (Scartabelli et al., 2008).

Metabotropic glutamate receptors (mGluRs) of group I (mGluR1 and mGluR5) are specifically localized at preand post-synaptic sites in both neurons, glial and microglial cells (Niswender and Conn, 2010; R. et al., 2013). It was previously reported that neurotoxicity induced by OGD is most likely mediated by mGlu1 rather than mGlu5 receptors in organotypic hippocampal slices (Pellegrini-Giampietro, 2003). On the other hand, we observed that DHPG Pre- and PostC induces ischemic tolerance in the same model via the contribution of both mGlu1 and mGlu5 receptors (Werner et al., 2007; Scartabelli et al., 2008). Therefore, a recent paper of our lab have shown that low, subtoxic concentrations of DHPG $(1 \mu \mathrm{M})$ could also prevent the loss of neurons induced by OGD leading to neuroprotection via mGluR5 (Cavallo et al., 2020). On the contrary, astrocytes were not significantly affected by DHPG (Cavallo et al., 2020) or DHPG PostC (Supplementary data, Fig. 2 panel A). Moreover, in OGD slices treated with DHPG, microglia cells display a morphology typical of reactive microglial (Cavallo et al., 2020), that in DHPG PostC appear to mediate ischemic tolerance by enhancing the protective phenotype M2 without changing the total amount of microglia cells (Supplementary data, Fig. 2 panel B and C).

In this study, we firstly show that DHPG PostC induces neuroprotection against OGD by decreasing the formation of ROS thought Akt activation in organotypic hippocampal slices (Fig. $1 \mathrm{~A}-\mathrm{C}$ ). This reduction is similar to what obtained by the classical ROS scavenger resveratrol, that is also able to induce neuroprotection when used in the same experimental condition of DHPG PostC (Supplementary data, Fig. 1).

The PI3K/Akt signaling pathway plays a central role in regulating cell growth, proliferation and survival under physiologic and pathophysiologic conditions (Cantley, 2002). Gao and colleagues have demonstrated that ischemic PostC enhances the phosphorylation of Akt and that the tolerance induced by PostC in stroke is partly abolished by the inhibition of Akt activity (Gao et al., 2008). It is well known that activation of PI3K is important in the intracellular signaling leading to cardioprotection and neuroprotection by ischemic or pharmacological PostC both in in vitro and in vivo models ( $\mathrm{Li}$ and Zuo, 2011; Wang et al., 2010). Many neuroprotectants, including propofol (Wang et al., 2009), estradiol and progesterone (Perez-Alvarez et al., 2015), N-acetyl serotonin (Yoo et al., 2017), thyroid hormone metabolite (Landucci et al., 2019; Laurino et al., 2018), manifest their protective effect through the PI3K/Akt pathway. Downstream targets of PI3K in PostC include the phosphorylation of components of the PI3K/Akt and of GSK3 $\beta$ (Zhou et al., 2011; Qi et al., 2012). Our results show that GSK3 $\beta$ phosphorylation and its subsequent inactivation is necessary for neuroprotection associated with DHPG PostC and the inhibitors of GSK3 $\beta$ are neuroprotective in our OGD model (Fig. 5A and B). The GSK3 $\beta$ inhibitor could also partially protect neurons against glutamate-induced excitotoxicity in primary cultures of rat cerebellar granule neurons (Hu et al., 2013). Moreover, in our model the GSK3 $\beta$ inhibitors TC-G 24 and $\mathrm{LiCl}$ are neuroprotective against OGD and could be used as PostC agents (Fig. 5C). Similar results were observed also in in vivo models of cerebral ischemia in which lithium improves functional and behavioral outcome in gerbils subjected to two vessels occlusion (Bian et al., 2007) and in rats subjected to MCAO (Taliyan and Ramagiri, 2016). Lithium is able to attenuate OGD injury only in combination with 4phenylbutirric acid in SK-N-MC neuronal cell cultures (Tung et al., 2015) and does not afford protection in adult hippocampal slices (Rosa et al., 2008).

GSK3 $\beta$ could have a central part in the regulation of $\beta$ catenin activation and translocation from cytoplasm to the nucleus, where $\beta$-catenin acts as a transcriptional coactivator, activating genes involved in cell survival and neuroprotection. In a recent paper of Tang and collaborators was observed that following global ischemia a variety of Wnt-related proteins, including Wnt and $\beta$-catenin (proteins involved in neuroprotection), were significantly reduced, meanwhile, GSK-3 $\beta$, which promotes negative effects, was significantly increased (Tang et al., 2019). The Wnt/ $\beta$-catenin signaling pathway
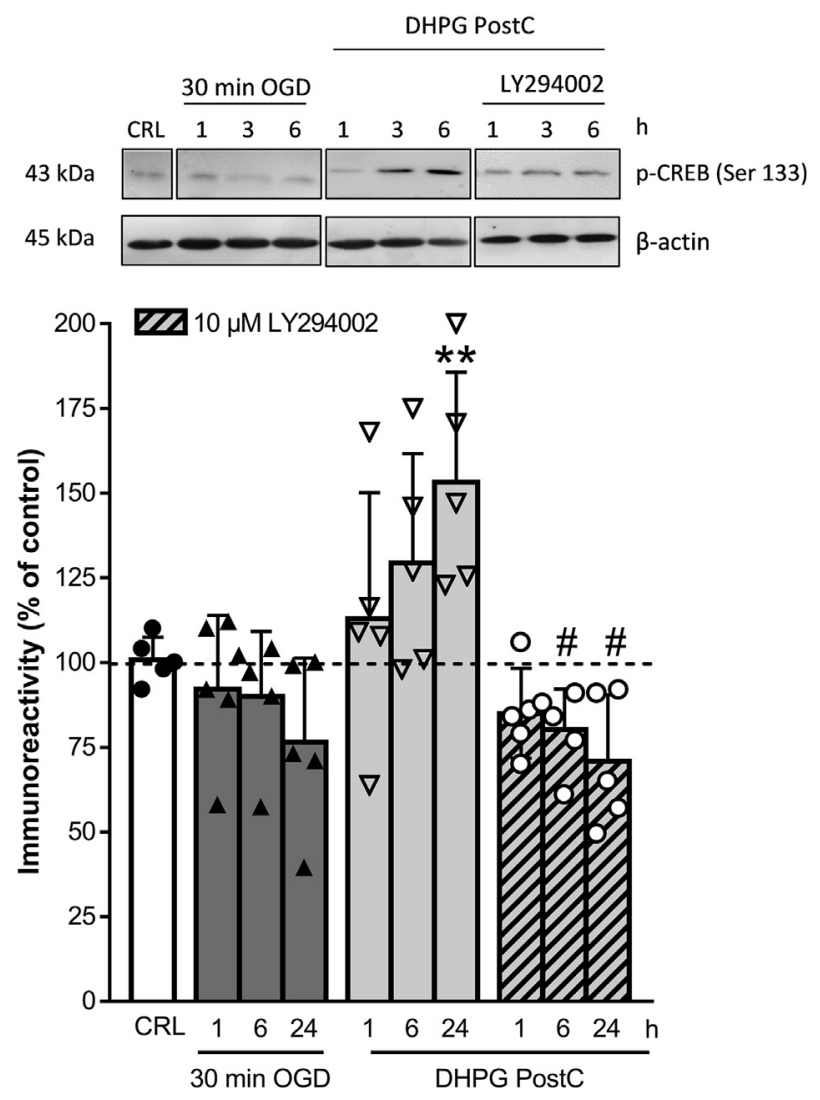

Fig. 4. DHPG PostC induces an increase in CREB phosphorylation. 1,6 or $24 \mathrm{~h}$ after treatments, the levels of CREB phosphorylation was evaluated through Western blot analysis. Upper panel: representative image for phosphorylated CREB ( $p$-CREB). Lower panel: quantitative analysis of showing that DHPG PostC induced an increase in CREB phosphorylation that was significantly decreased in presence of the Akt inhibitor LY294002. Data are expressed as percentage of CREB phosphorylation in control slices. Bars represent the mean \pm SEM of at least five experiments. ${ }^{* *} p<0.01$ vs. CRL, ${ }^{\#} p<0.05$ vs. $24 \mathrm{~h}$ DHPG PostC (ANOVA + Tukey's $w$ test). 
A

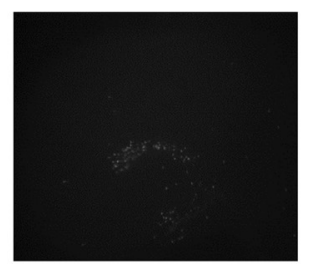

CRL

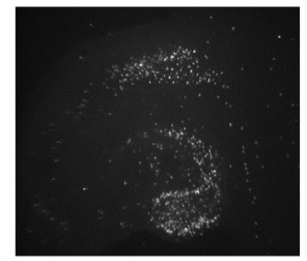

$\mathrm{OGD}+\mathrm{TC}-\mathrm{G}-24$

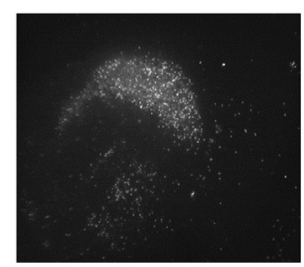

OGD+TC-G-24 PostC

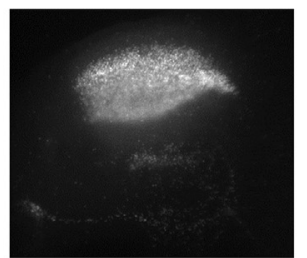

OGD

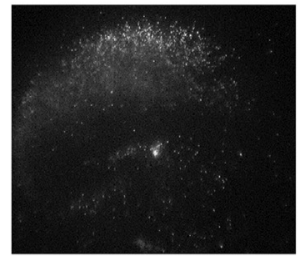

$\mathrm{OGD}+\mathrm{LiCl}$

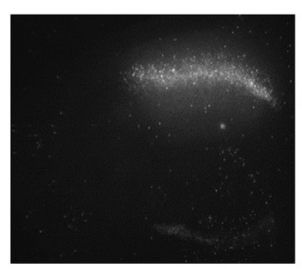

OGD + LiCl PostC
B
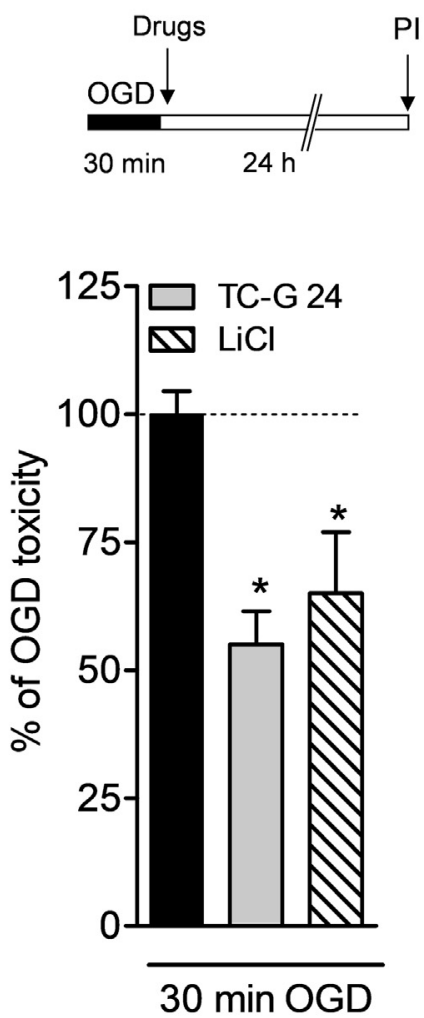

C
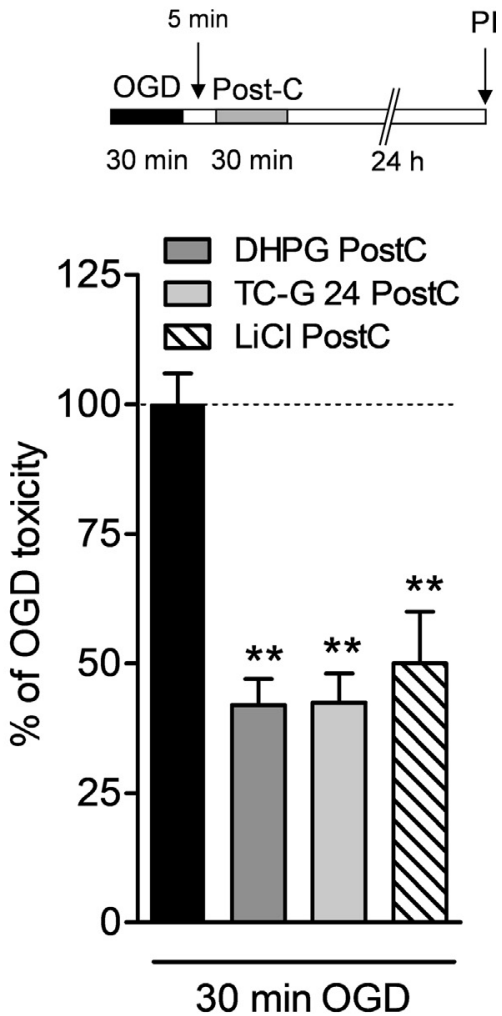

Fig. 5. GS3K inhibitors attenuates neurotoxicity and are involved in the induction of OGD tolerance. (A) Control: displaying background levels of PI fluorescence, OGD: displaying an intense PI labeling in CA1 region $24 \mathrm{~h}$ later. Slices incubated with TC-G 24 or LiCl after OGD or used as PostC show a reduction of CA1 PI fluorescence (B) Upper panel showing the experimental protocol. Lower panel showing that incubation with the GS3K inhibitors TC-G 24 and LiCl significantly attenuated OGD injury (quantitative analysis). (C) Upper panel showing the experimental protocol. Lower panel showing that slices exposed to DHPG or to TC-G 24 or LiCl PostC significantly attenuated OGD injury (quantitative analysis). Values represent the mean \pm SEM of at least three experiments performed in quadruplicate. ${ }^{*} p<0.05$ and ${ }^{* *} p<0.01$ vs. 30 min OGD alone (ANOVA + Tukey's $w$ test).

was downregulating by hypoxia-ischemia in $\mathrm{H} 9 \mathrm{C} 2$ cells (Tao et al., 2016) and its dysfunction in temporal lobe epilepsy is responsible of structural and functional abnormalities, while restoring Wnt pathway leads to neuroprotective effects (Huang et al., 2015). Similarly, the neuroprotective effects of isoflurane PostC is mediated by $W n t / \beta$-catenin signaling in MCAO models of cerebral ischemia in rats (Zhang et al., 2019).

In our DHPG PostC model, we also observed accumulation of $\beta$-catenin in the cytoplasm and translocation from the cytosol to nucleus (Fig. 3), which were dependent on PI3K/Akt/GSK3 $\beta$ activity.

The activation of the Wnt pathway through different ligands, such as lithium, induced an increase in intracellular calcium levels that trigger a sequence of events that include CREB phosphorylation and transcription of CREB-dependent genes that promote survival (Arrázola et al., 2009). In accordance, DHPG PostC induced an increase in CREB phosphorylation in a time dependent manner and Akt inhibitors reverted this effect (Fig. 4).

In conclusion, our results show that PostC induced neuroprotection by the activation of $\mathrm{PI} 3 \mathrm{~K} / \mathrm{Akt} / \mathrm{GSK} 3 \beta$ pro-survival pathway. In addition, GSK3 $\beta$ is not only a downstream target of this pathway, but is also involved in the Wnt pathway and its inactivation leads to $\beta$ catenin accumulation and translocation from the cytosol into the nucleus, that ultimately leading to CREB phosphorylation and transcription of CREB-dependent genes that promote survival.

\section{ACKNOWLEDGEMENTS}

This work was supported by the Ente Cassa di Risparmio di Firenze (Florence, Italy).

\section{REFERENCES}

Arrázola MS, Varela-Nallar L, Colombres M, Toledo EM, Cruzat F, Pavez L, Assar R, Aravena A, González M, Montecino M, Maass A, Martínez S, Inestrosa NC (2009) Calcium/calmodulindependent protein kinase type IV Is a target gene of the Wnt/ $\beta$ catenin signaling pathway. J Cell Physiol. https://doi.org/10.1002/ icp.21902.

Bian Q, Shi T, Chuang DM, Qian Y (2007) Lithium reduces ischemiainduced hippocampal CA1 damage and behavioral deficits in gerbils. Brain Res. https://doi.org/10.1016/j.brainres.2007.09.054.

Boscia F, Annunziato L, Taglialatela M (2006) Retigabine and flupirtine exert neuroprotective actions in organotypic 
hippocampal cultures. Neuropharmacology. https://doi.org/ 10.1016/j.neuropharm.2006.03.024.

Cantley LC (2002) The phosphoinositide 3-kinase pathway. Science. 0; 0. https://doi.org/10.1126/science.296.5573.1655.

Cavallo D, Landucci E, Gerace E, Lana D, Ugolini F, Henley JM, Giovannini MG, Pellegrini-Giampietro DE (2020) Neuroprotective effects of mGluR5 activation through the PI3K/Akt pathway and the molecular switch of AMPA receptors. Neuropharmacology 162. https://doi.org/10.1016/j.neuropharm.2019.107810.

Cook D, Fry MJ, Hughes K, Sumathipala R, Woodgett JR, Dale TC (1996) Wingless inactivates glycogen synthase kinase-3 via an intracellular signalling pathway which involves a protein kinase $C$. EMBO J. https://doi.org/10.1002/j.1460-2075.1996.tb00830.x.

Ding VW, Chen RH, McCormick F (2000) Differential regulation of glycogen synthase kinase $3 \beta$ by insulin and Wnt signaling. J Biol Chem. https://doi.org/10.1074/jbc.M005342200.

Gallin WJ, Greenberg ME (1995) Calcium regulation of gene expression in neurons: the mode of entry matters. Curr Opin Neurobiol. https://doi.org/10.1016/0959-4388(95)80050-6.

Gao X, Zhang H, Takahashi T, Hsieh J, Liao J, Steinberg GK, Zhao H (2008) The Akt signaling pathway contributes to postconditioning's protection against stroke; the protection is associated with the MAPK and PKC pathways. J Neurochem. https://doi.org/10.1111/j.1471-4159.2008.05218.x.

Gerace E, Landucci E, Scartabelli T, Moroni F, Chiarugi A, PellegriniGiampietro DE (2015) Interplay between histone acetylation/ deacetylation and poly(ADP-ribosyl)ation in the development of ischemic tolerance in vitro. Neuropharmacology. https://doi.org/ 10.1016/j.neuropharm.2015.01.008.

Gerace E, Landucci E, Scartabelli T, Moroni F, Pellegrini-Giampietro DE (2012a) Rat hippocampal slice culture models for the evaluation of neuroprotective agents. Methods Mol Biol. https:/l doi.org/10.1007/978-1-61779-536-7_29.

Gerace E, Masi A, Resta F, Felici R, Landucci E, Mello T, PellegriniGiampietro DE, Mannaioni G, Moroni F (2014) PARP-1 activation causes neuronal death in the hippocampal CA1 region by increasing the expression of $\mathrm{Ca} 2+$-permeable AMPA receptors. Neurobiol Dis. https://doi.org/10.1016/j.nbd.2014.05.023.

Gerace E, Scartabelli T, Formentini L, Landucci E, Moroni F, Chiarugi A, Pellegrini-Giampietro DE (2012b) Mild activation of poly(ADPribose) polymerase (PARP) is neuroprotective in rat hippocampal slice models of ischemic tolerance. Eur J Neurosci. https://doi.org/ 10.1111/j.1460-9568.2012.08116.x.

Gordon MD, Nusse R (2006) Wht signaling: Multiple pathways, multiple receptors, and multiple transcription factors. J Biol Chem. https://doi.org/10.1074/jbc.R600015200.

Guo JY, Yang T, Sun XG, Zhou NY, Li FS, Long D, Lin T, Li PY, Feng $L$ (2011) Ischemic postconditioning attenuates liver warm ischemia-reperfusion injury through Akt-eNOS-NO-HIF pathway. J Biomed Sci. https://doi.org/10.1186/1423-0127-18-79.

Hu S, Cui W, Mak S, Tang J, Choi C, Pang Y, Han Y (2013) Bis (propyl)-cognitin protects against glutamate-induced neuroexcitotoxicity via concurrent regulation of NO, MAPK/ERK and PI3-K/Akt/GSK3 $\beta$ pathways. Neurochem Int. https://doi.org/ 10.1016/j.neuint.2013.01.

Huang C, Fu XH, Zhou D, Li JM (2015) The role of Wnt/ß-catenin signaling pathway in disrupted hippocampal neurogenesis of temporal lobe epilepsy: a potential therapeutic target? Neurochem Res. https://doi.org/10.1007/s11064-015-1614-1.

Landucci E, Gencarelli M, Mazzantini C, Laurino A, PellegriniGiampietro DE, Raimondi L (2019) N-(3-ethoxy-phenyl)-4pyrrolidin-1-yl-3-trifluoromethyl-benzamide (EPPTB) prevents 3iodothyronamine (T1AM)-induced neuroprotection against kainic acid toxicity. Neurochem Int 129. https://doi.org/10.1016/i. neuint.2019.05.004 104460.

Landucci E, Lattanzi R, Gerace E, Scartabelli T, Balboni G, Negri L, Pellegrini-Giampietro DE (2016) Prokineticins are neuroprotective in models of cerebral ischemia and ischemic tolerance in vitro. Neuropharmacology 108:39-48. https://doi.org/10.1016/j. neuropharm.2016.04.043.
Laurino A, Landucci E, Resta F, De Siena G, Pellegrini-Giampietro DE, Masi A, Mannaioni G, Raimondi L (2018) Anticonvulsant and neuroprotective effects of the thyroid hormone metabolite 3iodothyroacetic acid. Thyroid 28:1387-1397. https://doi.org/ 10.1089/thy.2017.0506.

$\mathrm{Li}$ L, Zuo Z (2011) Isoflurane postconditioning induces neuroprotection via Akt activation and attenuation of increased mitochondrial membrane permeability. Neuroscience 199:44-50.

Liang MH, Chuang DM (2007) Regulation and function of glycogen synthase kinase-3 isoforms in neuronal survival. J Biol Chem. https://doi.org/10.1074/jbc.M605178200.

Liu R, Liu W, Doctrow SR, Baudry M (2003) Iron toxicity in organotypic cultures of hippocampal slices: Role of reactive oxygen species. J Neurochem. https://doi.org/10.1046/j.14714159.2003.01708.x.

Nie H, Xiong L, Lao N, Chen S, Xu N, Zhu Z (2006) Hyperbaric oxygen preconditioning induces tolerance against spinal cord ischemia by upregulation of antioxidant enzymes in rabbits. $J$ Cereb Blood Flow Metab. https://doi.org/10.1038/sj.jcbfm. $\underline{9600221 .}$

Niswender CM, Conn PJ (2010) Metabotropic glutamate receptors: physiology, pharmacology, and disease. Annu Rev Pharmacol Toxicol 50:295-322. https://doi.org/10.1146/annurev. pharmtox. 011008.145533.

Pellegrini-Giampietro DE (2003) The distinct role of mGlu1 receptors in post-ischemic neuronal death. Trends Pharmacol Sci. https:// doi.org/10.1016/S0165-6147(03)00231-1.

Perez-Alvarez MJ, Mateos L, Alonso A, Wandosell F (2015) Estradiol and progesterone administration after pMCAO stimulates the neurological recovery and reduces the detrimental effect of ischemia mainly in hippocampus. Mol Neurobiol. https://doi.org/ 10.1007/s12035-014-8963-7.

Pignataro G, Meller R, Inoue K, Ordonez AN, Ashley MD, Xiong Z, Simon RP (2008) In vivo and in vitro characterization of a novel neuroprotective strategy for stroke: Ischemic postconditioning. J Cereb Blood Flow Metab. https://doi.org/10.1038/sj.jcbfm. $\underline{9600559}$.

Qi ZF, Luo YM, Liu XR, Wang RL, Zhao HP, Yan F, Song ZJ, Luo M, Ji XM (2012) AKT/GSK3 $\beta$-dependent autophagy contributes to the neuroprotection of limb remote ischemic postconditioning in the transient cerebral ischemic rat model. CNS Neurosci Ther. https://doi.org/10.1111/cns.12016.

Roel K, De Vries HE, Geurts JJG (2013) Grey matter damage in multiple sclerosis: a pathology perspective. Prion 7:66-75.

Ren C, Gao X, Niu G, Yan Z, Chen X, Zhao H (2008) Delayed postconditioning protects against focal ischemic brain injury in rats. PLoS ONE. https://doi.org/10.1371/journal.pone.0003851.

Rosa AO, Egea J, Martínez A, García AG, López MG (2008) Neuroprotective effect of the new thiadiazolidinone NP00111 against oxygen-glucose deprivation in rat hippocampal slices: Implication of ERK1/2 and PPAR $\gamma$ receptors. Exp Neurol. https:// doi.org/10.1016/j.expneurol.2008.03.008.

Scartabelli T, Gerace E, Landucci E, Moroni F, Pellegrini-Giampietro DE (2008) Neuroprotection by group I mGlu receptors in a rat hippocampal slice model of cerebral ischemia is associated with the PI3K-Akt signaling pathway: a novel postconditioning strategy? Neuropharmacology 55:509-516. https://doi.org/ 10.1016/j.neuropharm.2008.06.019.

Song W, Sun J, Su B, Yang R, Dong H, Xiong L (2013) Ischemic postconditioning protects the spinal cord from ischemiareperfusion injury via modulation of redox signaling. $\mathrm{J}$ Thorac Cardiovasc Surg. https://doi.org/10.1016/i.jtcvs.2012.11.039.

Taliyan R, Ramagiri S (2016) Delayed neuroprotection against cerebral ischemia reperfusion injury: putative role of BDNF and GSK-3ß. J. Recept. Signal Transduct.. https://doi.org/10.3109/ 10799893.2015.1108338.

Tang Y, Shen J, Zhang F, Yang FY, Liu M (2019) Human serum albumin attenuates global cerebral ischemia/reperfusion-induced brain injury in a Wnt/ $\beta$-Catenin/ROS signaling-dependent manner in rats. Biomed Pharmacother. https://doi.org/10.1016/i. biopha.2019.108871. 
Tao J, Abudoukelimu M, Ma YT, Yang YN, Li XM, Chen BD, Liu F, He $\mathrm{CH}$, Li HY (2016) Secreted frizzled related protein 1 protects H9C2 cells from hypoxia/re-oxygenation injury by blocking the Wnt signaling pathway. Lipids Health Dis. https://doi.org/10.1186/ s12944-016-0240-5.

Tsutsumi YM, Yokoyama T, Horikawa Y, Roth DM, Patel HH (2007) Reactive oxygen species trigger ischemic and pharmacological postconditioning: In vivo and in vitro characterization. Life Sci. https://doi.org/10.1016/j.Ifs.2007.08.031.

Tung WF, Chen WJ, Hung HC, Liu GY, Tung JN, Huang CC, Lin CL (2015) 4-Phenylbutyric acid (4-PBA) and lithium cooperatively attenuate cell death during oxygen-glucose deprivation (OGD) and reoxygenation. Cell Mol Neurobiol. https://doi.org/10.1007/ s10571-015-0179-5.

Wang HY, Wang GL, Yu YH, Wang Y (2009) The role of phosphoinositide-3-kinase/Akt pathway in propofol-induced postconditioning against focal cerebral ischemia-reperfusion injury in rats. Brain Res. https://doi.org/10.1016/j.brainres.2009. $\underline{08.054}$.

Wang JK, Yu LN, Zhang FJ, Yang MJ, Yu J, Yan M, Chen G (2010) Postconditioning with sevoflurane protects against focal cerebral ischemia and reperfusion injury via PI3K/Akt pathway. Brain Res. https://doi.org/10.1016/j.brainres.2010.08.009.

Wang JY, Shen J, Gao Q, Ye ZG, Yang SY, Liang HW, Bruce IC, Luo $\mathrm{BY}$, Xia $Q$ (2008) Ischemic postconditioning protects against global cerebral ischemia/reperfusion-induced injury in rats. Stroke. https://doi.org/10.1161/STROKEAHA.107.499079.

Wang Q, Zhang X, Ding Q, Hu B, Xie Y, Li X, Yang Q, Xiong L (2011) Limb remote postconditioning alleviates cerebral reperfusion injury through reactive oxygen species-mediated inhibition of delta protein kinase $\mathrm{C}$ in rats. Anesth Analg. https://doi.org/ 10.1213/ANE.0b013e31822b885f.

Werner CG, Scartabelli T, Pancani T, Landucci E, Moroni F, Pellegrini-Giampietro DE (2007) Differential role of mGlu1 and mGlu5 receptors in rat hippocampal slice models of ischemic tolerance. Eur J Neurosci 25:3597-3604. https://doi.org/10.1111/ j.1460-9568.2007.05614.X.

Xing B, Chen H, Zhang M, Zhao D, Jiang R, Liu X, Zhang S (2008a) Ischemic postconditioning inhibits apoptosis after focal cerebral ischemia/reperfusion injury in the rat. Stroke. https://doi.org/ 10.1161/STROKEAHA.107.507939.

Xing B, Chen H, Zhang M, Zhao D, Jiang R, Liu X, Zhang S (2008b) Ischemic post-conditioning protects brain and reduces inflammation in a rat model of focal cerebral ischemia/ reperfusion. J Neurochem. https://doi.org/10.1111/j.1471-4159. 2008.05276.X.

Ye Z, Guo Q, Xia P, Wang N, Wang E, Yuan Y (2012) Sevoflurane postconditioning involves an up-regulation of HIF-1 $\alpha$ and $\mathrm{HO}-1$ expression via PI3K/Akt pathway in a rat model of focal cerebral ischemia. Brain Res. https://doi.org/10.1016/j.brainres.2012. 04.050 .

Yoo JM, Lee BD, Sok DE, Ma JY, Kim MR (2017) Neuroprotective action of $\mathrm{N}$-acetyl serotonin in oxidative stress-induced apoptosis through the activation of both TrkB/CREB/BDNF pathway and Akt/Nrf2/Antioxidant enzyme in neuronal cells. Redox Biol. https:// doi.org/10.1016/j.redox.2016.12.034.

Zhang G, Ge M, Han Z, Wang S, Yin J, Peng L, Xu F, Zhang Q, Dai Z, Xie L, Li Y, Si J, Ma K (2019) Wnt//-catenin signaling pathway contributes to isoflurane postconditioning against cerebral ischemia-reperfusion injury and is possibly related to the transforming growth factor $\beta 1 / \mathrm{Smad} 3$ signaling pathway. Biomed Pharmacother. https://doi.org/10.1016/j.biopha.2018.11.143.

Zhao H, Ren C, Chen X, Shen J (2012) From rapid to delayed and remote postconditioning: the evolving concept of ischemic postconditioning in brain ischemia. Curr Drugs Targets. https:// doi.org/10.2174/138945012799201621.

Zhou C, Tu J, Zhang Q, Lu D, Zhu Y, Zhang W, Yang F, Brann DW, Wang R (2011) Delayed ischemic postconditioning protects hippocampal CA1 neurons by preserving mitochondrial integrity via Akt/GSK3 $\beta$ signaling. Neurochem Int. https://doi.org/10.1016/ j.neuint.2011.08.008.

\section{APPENDIX A. SUPPLEMENTARY DATA}

Supplementary data to this article can be found online at https://doi.org/10.1016/j. neuroscience.2019.12.047. 